\title{
Presentación
}

\section{Educaciones emancipadoras de Nuestra América. Sujetos y procesos}

Desde hace décadas se ha desarrollado en Latinoamérica una serie de prácticas, experiencias, reflexiones e investigaciones educativas contrahegemónicas que plantean propuestas y críticas al modelo de educación moderno que se implementó a lo largo de dos siglos en el continente. Estas acciones educativas tensan los principios de la modernidad capitalista, principios que se han asumido como valores y saberes fundamentales en los procesos formativos que implementan los Estados.

Para las educaciones contrahegemónicas, el capitalismo encontró en la modernidad un aliado central para fortalecer los principios y rasgos característicos que le permitieron anclarse socialmente. El elogio a la individualidad, a la urbanización, a la razón, al consumismo, al progreso, son rasgos que la modernidad y el capitalismo han convertido en principios máximos para la vida. Esta convergencia es lo que Bolívar Echeverría ha denominado modernidad capitalista.

A contrapelo de la modernidad capitalista y proponiendo otros principios y rasgos, emergen y se actualizan con fuerza procesos educativos emancipadores en América Latina. Se trata de propuestas, reflexiones e investigaciones que plantean intencionalidades sociopolíticas y culturales como la construcción de un mundo más equitativo que reconozca y valore la pluralidad, un mundo en el que quepan muchos mundos. Experiencias como la Educación del Campo en Brasil, la educación popular, la educación intercultural, la educación autónoma, 
la educación propia, la educación crítica desde la perspectiva latinoamericana, entre otras, han sido construidas por sujetos histórico-políticos, por colectivos sociales que han configurado sentidos, prácticas, teorías, discursos, intenciones, pedagogías y formas de hacer la educación desde perspectivas plurales que cuestionan la educación moderna que se mantiene en la sociedad.

Estas experiencias educativas se han desarrollado bajo principios pedagógicos, organizativos y políticos, como también bajo rasgos característicos que reconocen que los procesos formativos requieren situar lo educativo en su dimensión política; en este caso, con una intencionalidad emancipatoria y de liberación humana. Para ello, es fundamental que el proyecto educativo establezca el vínculo dialéctico con un sujeto pedagógico y con un método que le permita una lectura crítica de la realidad social, con la reivindicación del derecho colectivo a la escuela, al conocimiento y una praxis político-pedagógica para la transformación social.

Por ejemplo, el reconocimiento de conocimientos distintos a los avalados por las comunidades científicas; un currículo que refleje la cultura, los valores, los saberes, las identidades y los modos de vida de la comunidad; la inclusión en la escuela de prácticas sociales que potencian la vida colectiva; la relación profunda entre la escuela y la vida cotidiana; la valoración de la vida rural o del campo como propuesta para la soberanía alimentaria; el vínculo y valoración de los abuelos como transmisores del legado ancestral; el respeto y la lucha por el territorio, entre otras, son prácticas educativas que están fisurando los ideales hegemónicos de educación porque ponen el acento en el conocimiento de la vida cotidiana y en una pedagogía de la lucha social.

Los procesos educativos emancipadores de Nuestra América tienen intencionalidades profundas en la formación de las personas. En general se pretende que los sujetos transiten otros caminos, se piensen desde lógicas distintas a las que viven, "retornen" a los proyectos colectivos y culturales, potencien los procesos socio políticos que están en marcha. Es decir, hay intencionalidades explícitas que van más allá de la formación para el conocimiento y se transita hacia la formación para la transformación.

Con base en lo anterior, este número temático de la Revista Colombiana de Educación, bajo el título "Educaciones Emancipadoras de Nuestra América. Sujetos y procesos", reúne una antología de artículos que busca dar un lugar a las propuestas educativas que están transitando por varios países de Latinoamérica. Destacamos nuestra alegría por recibir una expresiva cantidad de propuestas que reflejan el amplio interés en la reflexión relacionada a la dimensión emancipadora de la educación. Asimismo, señalamos que, en virtud de ello, tenemos dos números de esa edición temática. 
En ese sentido, lo que pretendemos es dar a conocer las historias de diferentes procesos sociopolíticos de América Latina que potencian, a través de lo educativo, la lucha por el reconocimiento de otras pedagogías, la revitalización de otras formas de pensamiento, el fortalecimiento del territorio, la construcción de una concepción de educación hacia la emancipación. Además de hacer evidentes las tensiones, las disputas y los problemas sociales, políticos, pedagógicos y culturales que han tenido que enfrentar estos procesos educativos al tratar de construir y mantener su perspectiva contrahegemónica.

Así, presentamos los textos que componen ese dosier, los cuales traen resultados de lecturas y de investigaciones realizadas por académicos y educadores de algunas universidades de Nuestra América. Los dos primeros artículos presentan discusiones acerca de perspectivas de las educaciones emancipatorias, y a partir del tercer artículo, se abordan experiencias y análisis de procesos educativos emancipatorios de la historia reciente del continente latinoamericano.

El artículo que abre el dosier, intitulado "Pedagogías Sentipensantes y Revolucionarias en la praxis educativo-política de los movimientos sociales de América Latina", de Lia Pinheiro Barbosa, plantea algunos elementos de carácter teórico y metodológico para pensar el campo problemático que articula la relación entre procesos históricos de resistencia, la educación y el conocimiento en la conformación de Pedagogías Sentipensantes y Revolucionarias de la praxis educativo-política de los movimientos indígenas y campesinos de América Latina. La autora argumenta que estas Pedagogías se nutren de fundamentos de orden histórico-político que configuran la subjetividad política y la concepción de horizonte emancipatorio, a la vez que enfatiza, que la concepción de educación construida por los movimientos indígenas y campesinos está vinculada a una matriz ontológica, epistémica e identitaria de sus territorios y del Abya Yala. De esa forma, existe tanto un fortalecimiento de otras racionalidades que construyen un paradigma epistémico intitulado Paradigma Epistémico del Campo como del proceso de formación de un sujeto histórico-político.

El segundo artículo, titulado "La ruta de Paulo Freire en Chile (19641969). Alfabetización popular, influencias teóricas y desplazamientos político-pedagógicos", de Fabian Cabaluz y Beatríz Areyuna, se sitúa en el campo de la historia de la educación y profundiza en la experiencia del exilio vivida por el pedagogo brasilero Paulo Freire en Chile durante los años 1964 y 1969. Se centra en analizar la participación del pedagogo brasileño en las campañas de alfabetización popular del gobierno de Eduardo Frei Montalva y plantea, a modo de hipótesis, que la experiencia del exilio de Freire en Chile contribuyó a que su obra alimentara y se nutriera los procesos de radicalización política vividos en el país durante 
la década de los sesenta, lo que en términos teóricos se expresó en la incorporación de autores y planteamientos asociados a un marxismo heterodoxo y crítico.

El tercer artículo que abre paso a las experiencias se titula "La segmentación territorial de la educación general básica y el pueblo indígena kichwa kisapincha de Ambato - Tungurahua- Ecuador", de Judith Pinos Montenegro. En el contexto ecuatoriano, la autora analiza cómo ha sido la recepción, por parte de los agentes indígenas del pueblo kichwa kisapincha de Ambato-Tungurahua-, Ecuador, de la política pública de segmentación territorial de la educación general básica, que rige desde el año 2011. Para ello, la autora realizó un estudio de caso durante los años 2017 a 2019. Los hallazgos principales muestran que, en el año 2017, la segmentación territorial de los servicios escolares fue percibida como una arbitrariedad del Estado, que incumplió con el mandato constitucional de consulta previa y afectó el acceso a la educación. Sin embargo, la política pública es parcialmente aceptada y lo que se cuestiona es la poca pertinencia cultural de los servicios educativos.

Para trasladar nuestra mirada al contexto de Colombia, el cuarto artículo denominado "Educación popular y educación propia. Diálogos desde experiencias educativas en Cauca", de María Isabel González Terreros y Alfonso Torres Carrillo, presenta los diálogos entre la educación popular y la educación propia desde discursos y experiencias pedagógicas que han llevado a cabo profesores y dirigentes sociales del departamento del Cauca, Colombia. Los autores analizan las dos corrientes pedagógicas que hacen parte de las apuestas político - educativas de dos organizaciones que conviven en el Cauca: la Asociación de Instructores y Trabajadores de la Educación del Departamento del Cauca (Asoinca) y el Consejo Regional Indígena del Cauca (CRIC). En su análisis, consideran que, a pesar de las perspectivas diferentes, existe un diálogo de sentidos y saberes entre las dos corrientes pedagógicas, el cual ha posibilitado construcciones colectivas en algunas prácticas escolares donde convergen.

El quinto artículo: "Educación campesina: las dimensiones pedagógico-políticas en la formación de la CLOC-VC y la ust como experiencia de lucha", de Oscar Humberto Soto, analiza el contexto de conformación de un proyecto educativo campesino que se articula a la Coordinadora Latinoamericana de Organizaciones del Campo (CLOC), de la Vía Campesina (vc), con especial atención en la experiencia de resistencia de la Unión de Trabajadores Rurales Sin Tierra (UST) en Argentina, entre los años 2010 y 2019. El autor analiza el carácter político de las prácticas campesinas en las que los proyectos pedagógicos afloran como horizonte de sentido. Este trabajo es abordado desde una metodología de investigación-militante 
y situada, con el objeto de registrar la potencialidad transformadora de los movimientos sociales del mundo rural a partir de sus propuestas de educación y formación campesina.

El último artículo del primer número temático se titula "La educación escolar quilombola en la perspectiva del hombre del campo: lo Camponês Quilombola", de las autoras Niltânia Brito Oliveira y Arlete Ramos dos Santos. Este analiza las políticas educativas del Plano de Acciones Articuladas (PAR) en algunas municipalidades de Bahía/Brasil en el contexto de la Educación de Campo. Para ello, las autoras destacan la experiencia educativa escolar de los quilombolas, reconociéndoles como sujetos campesinos. Al respecto plantean que ellos hacen parte de la clase campesina y por esa razón proponen la categoría campesino quilombola, que representa a los trabajadores rurales que viven en los quilombos con sus modos específicos de producción, conocimientos e historias que entran en disputa con el modo capitalista de producción. A partir de los resultados de la investigación realizada en los documentos oficiales se constata que hay contradicciones entre las normativas de las legislaciones específicas y la materialidad de los hechos relacionados directamente con las políticas públicas hacia los quilombolas.

Agradecemos a los autores que enviaron sus artículos, especialmente a quienes hacen parte de este primer número del dosier que ofrece contribuciones de Brasil, Ecuador, Argentina, Chile y Colombia, ya que contribuyen al reconocimiento del pensamiento crítico. Este primer número recoge tanto discusiones sobre las educaciones emancipatorias como experiencias y prácticas educativas que hacen parte de procesos organizativos a lo largo del continente. Es para nosotras un gusto presentarles este dosier que, con seguridad, contribuirá a las prácticas educativas emancipadoras de Nuestra América.

María Isabel González Terreros

Universidad Pedagógica Nacional

Arlete Ramos dos Santos

Universidade Estadual do Sudoeste da Bahia

Lia Pinheiro Barbosa

Universidade Estadual do Ceará 